\title{
A Spectral Galerkin Method for a Boundary Integral Equation
}

\author{
By W. McLean
}

\begin{abstract}
We consider the boundary integral equation which arises when the Dirichlet problem in two dimensions is solved using a single-layer potential. A spectral Galerkin method is analyzed, suitable for the case of a smooth domain and smooth boundary data. The use of trigonometric polynomials rather than splines leads to fast convergence in Sobolev spaces of every order. As a result, there is rapid convergence of the approximate solution to the Dirichlet problem and all its derivatives uniformly up to the boundary.
\end{abstract}

1. Introduction. Let $\Omega$ denote a bounded open subset of $\mathbb{R}^{2}$ with boundary

$$
\Gamma=\partial \Omega \text {. }
$$

The interior and exterior Dirichlet problem is to find $U$ satisfying

$$
\begin{array}{ll}
\Delta U=0 & \text { on } \mathbb{R}^{2} \backslash \Gamma, \\
U=g & \text { on } \Gamma, \\
U(X)=\frac{\beta}{\pi} \log \left(\frac{1}{|X|}\right)+O(1) & \text { as }|X| \rightarrow \infty,
\end{array}
$$

where $g$ is a given function on $\Gamma$, and $\beta$ is a specified constant. A unique classical solution exists, assuming $\Gamma$ is Lipschitz and $g$ is continuous. We shall seek a function $v \in L_{2}(\Gamma)$ and a constant $\alpha$ such that this solution is given by the single-layer potential

$$
U(X)=\frac{1}{\pi} \int_{\Gamma} \log \left(\frac{1}{|X-Y|}\right) v(Y) d \sigma(Y)+\alpha, \quad X \in \mathbb{R}^{2},
$$

where $\sigma$ is the arclength measure on $\Gamma$. The formula (1.2) defines a function which is harmonic on $\mathbb{R}^{2} \backslash \Gamma$ and continuous on $\mathbb{R}^{2}$. The boundary condition (1.1b) is satisfied if and only if the pair $(v, \alpha)$ is a solution of the integral equation

$$
\frac{1}{\pi} \int_{\Gamma} \log \left(\frac{1}{|X-Y|}\right) v(Y) d \sigma(Y)+\alpha=g(X), \quad X \in \Gamma,
$$

and the required behavior at infinity (1.1c) occurs if and only if

$$
\int_{\Gamma} v d \sigma=\beta
$$

In order to state a basic existence and uniqueness theorem for (1.3), let $\mathbf{K}$ denote the real or complex number field and let $W_{p}^{1}(\Gamma)$ denote the Sobolev space consisting of those functions in $L_{p}(\Gamma)$ which have a weak tangential derivative in $L_{p}(\Gamma)$.

Received December 3, 1984.

1980 Mathematics Subject Classification. Primary 65R20; Secondary 65N30, 45L10, 42A10, 42A45. 
THEOREM 1.1 ([10]). If $\Gamma$ is Lipschitz, then the operator $(v, \alpha) \mapsto(g, \beta)$ defined by (1.3) is an isomorphism $L_{2}(\Gamma) \times \mathbb{K} \rightarrow W_{2}^{1}(\Gamma) \times \mathbb{K}$.

The proof of this result is straightforward, given the work of Verchota [16] on the classical method of layer potentials for Lipschitz domains.

From this point on, it will be assumed that $\Gamma$ is $C^{\infty}$, except where something to the contrary is stated explicitly. Moreover, we shall assume for convenience that $\Gamma$ is connected and $g \in C^{\infty}(\Gamma)$.

Numerical methods for solving the integral equation (1.3), or equations closely related to it, have been discussed by many authors, including [1], [3], [5]-[12], [17]. A Galerkin method using periodic splines was analyzed by Hsiao, Kopp, and Wendland [6], [7], who proved typical finite element error estimates with polynomial rates of convergence limited by the degree of the splines. Later, Arnold [1] introduced a Petrov-Galerkin method with periodic splines as trial functions and trigonometric polynomials as test functions. He proved error estimates for the resulting approximations to $(v, \alpha)$ in Sobolev norms of arbitrarily large negative order, and from these was able to establish polynomial rates of convergence of arbitrarily high order for the corresponding approximations to $U$, the error being measured in the $L_{\infty}$-norm on compact subsets of $\mathbb{R}^{2} \backslash \Gamma$. Furthermore, by use of the Hilbert space $X_{s, \varepsilon}$ (see Section 5 below), Arnold showed that if $\Gamma$ and $g$ are not only $C^{\infty}$ but also analytic, then exponential rates of convergence are attained.

In what follows, we shall present a spectral Galerkin method for solving (1.3), in which trigonometric polynomials are used both as test functions and as trial functions. The rates of convergence attained are the same as in the Petrov-Galerkin method of Arnold, but in our case the error estimates also hold in stronger norms, with the result that the approximations to $U$ converge exponentially fast uniformly up to the boundary. We mention that a related technique was discussed briefly in a paper of Henrici [5, pp. 292, 501]. Also, in a recent paper, Lamp, Schleicher, and Wendland [9] have independently studied a generalization of the method, which allows one to deal with a class of periodic, elliptic pseudodifferential equations, and thus have obtained some of our results as special cases. (They consider the spaces $H_{p}^{s}$ only for $p=2$.)

2. The Integral Equation on the Torus. The one-dimensional torus

$$
\mathbb{T}=\mathbb{R} / 2 \pi \mathbb{Z}
$$

will be identified as a set with the half-open interval $(-\pi, \pi]$ in the usual way. Let

$$
\gamma=\left(\gamma_{1}, \gamma_{2}\right): \mathbb{T} \rightarrow \Gamma
$$

be a $C^{\infty}$ parametrization of $\Gamma$ satisfying

$$
|\dot{\gamma}(t)| \neq 0 \text { for }-\pi<t \leqslant \pi,
$$

where $\dot{\gamma}$ denotes the derivative of $\gamma$. By putting

$$
u(t)=v[\gamma(t)]|\dot{\gamma}(t)|, \quad f(t)=g[\gamma(t)],
$$

the integral equation (1.3) can be rewritten as

$$
\begin{gathered}
\frac{1}{\pi} \int_{-\pi}^{\pi} \log \left(\frac{1}{|\gamma(x)-\gamma(t)|}\right) u(t) d t+\alpha=f(x), \quad-\pi<x \leqslant \pi, \\
\int_{-\pi}^{\pi} u(t) d t=\beta .
\end{gathered}
$$


In the special case where $\gamma$ is a circle of radius $r$, one can take

$$
\gamma(t)=r e^{i t}, \quad-\pi<t \leqslant \pi
$$

then

$$
|\gamma(x)-\gamma(t)|=2 r|\sin ((x-t) / 2)| .
$$

In the general case, we write (cf. [5, Eq. (2.8)], [6], [9])

$$
\frac{1}{\pi} \log \left(\frac{1}{|\gamma(x)-\gamma(t)|}\right)=\Lambda(x-t)+k(x, t) \text {, }
$$

where

$$
\Lambda(t)=\frac{1}{\pi} \log \left(\frac{1}{|\sin (t / 2)|}\right)
$$

is singular but

$$
k(x, t)= \begin{cases}\frac{1}{\pi} \log \left(\frac{|\sin ((x-t) / 2)|}{|\gamma(x)-\gamma(t)|}\right) & \text { for } x \neq t, \\ \frac{1}{\pi} \log \left(\frac{1 / 2}{|\dot{\gamma}(t)|}\right) & \text { for } x=t,\end{cases}
$$

satisfies

$$
k \in C^{\infty}\left(\mathbb{T}^{2}\right)
$$

Define the integral operators

$$
A u(x)=\Lambda * u(x)=\int_{-\pi}^{\pi} \Lambda(x-t) u(t) d t, \quad K u(x)=\int_{-\pi}^{\pi} k(x, t) u(t) d t
$$

then (2.3) may be written

$$
\begin{gathered}
(A+K) u+\alpha=f \quad \text { on } \mathbb{T}, \\
\int_{-\pi}^{\pi} u d t=\beta .
\end{gathered}
$$

In the next section we shall discuss the solvability of these equations using the Bessel potential spaces

$$
H_{p}^{s}(\mathbb{T}), \quad 1<p<\infty, s \in \mathbb{R},
$$

which are defined as follows (cf. Nikol'skii [15], Bergh and Löfström [2]): Denote the Fourier coefficients of $u$ by

$$
\hat{u}_{m}=\int_{\pi}^{\pi} e^{-i m t} u(t) d t, \quad m \in \mathbb{Z}
$$

define the basis functions

$$
e_{m}(t)=e^{i m t}, \quad m \in \mathbb{Z},
$$

and recall that for $1<p<\infty$, if $u \in L_{p}(\mathbb{T})$, the Fourier series

$$
u=\frac{1}{2 \pi} \sum_{m \in \mathbf{Z}} \hat{u}_{m} e_{m}
$$

converges in $L_{p}(\pi)$. Let $D=d / d t$ be the operator of differentiation in the sense of distributions on $\mathrm{T}$; then

$$
\left(D^{s} u\right)_{m}=(i m)^{s} \hat{u}_{m}, \quad s=0,1,2, \ldots
$$


For any real number $s$, put

$$
J^{s} u=\frac{1}{2 \pi} \sum_{m \in \mathbf{Z}}\left(1+|m|^{2}\right)^{s / 2} \hat{u}_{m} e_{m} ;
$$

then one can see from (2.6) that formally

$$
J^{s}=\left(1-D^{2}\right)^{s / 2}
$$

The space $H_{p}^{s}(\mathbb{T})$ consists of those distributions $u$ on $\mathbb{T}$ for which $J^{s} u \in L_{p}(\mathbb{T})$, and is a Banach space with norm $\|u\|_{H_{p}^{s}(\mathbf{T})}=\left\|J^{s} u\right\|_{p}$. The Bessel potential spaces are related to the familiar Sobolev spaces by

$$
H_{p}^{s}(\mathbb{T})=W_{p}^{s}(\mathbb{T}), \quad 1<p<\infty, s=0,1,2, \ldots,
$$

with equivalence of norms. When $p=2$, this fact is a consequence of Parseval's identity

$$
\|u\|_{2}=\frac{1}{2 \pi}\left(\sum_{m \in \mathbf{Z}}\left|\hat{u}_{m}\right|^{2}\right)^{1 / 2},
$$

and for general $p$ it can be proved using the following Fourier multiplier theorem.

TheOREM 2.1 (MARCinkiewicz [13]). Assume $1<p<\infty$. If the complex numbers $a_{m}, m \in \mathbb{Z}$, satisfy

$$
\begin{aligned}
& \left|a_{m}\right| \leqslant M \quad \text { for } m \in \mathbb{Z}, \\
& \sum_{2^{j} \leqslant|m|<2^{j+1}}\left|a_{m+1}-a_{m}\right| \leqslant M \quad \text { for } j=0,1,2, \ldots,
\end{aligned}
$$

then for every $u \in L_{p}(\mathbb{T})$ the sum $\sum_{m \in \mathbf{Z}} a_{m} \hat{u}_{m} e_{m}$ converges in $L_{p}(\mathbb{T})$ and there is a constant $c_{p}$ depending only on $p$ such that

$$
\left\|\sum_{m \in \mathbf{Z}} a_{m} \hat{u}_{m} e_{m}\right\|_{p} \leqslant c_{p} M\|u\|_{p} .
$$

3. Regularity Theory. Our aim in this section is to generalize Theorem 1.1 by determining the precise relation between the smoothness of the solution to the integral equation and the smoothness of the boundary data. The first step is to determine how $A$ transforms the Bessel potential spaces.

Observe that, since

$$
D \Lambda(t)=-\frac{1}{2 \pi} \operatorname{cotan}\left(\frac{t}{2}\right),
$$

the operator $A$ is related to the Hilbert transform

$$
H u(x)=\frac{1}{2 \pi i} f_{-\pi}^{\pi} \operatorname{cotan}\left(\frac{t-x}{2}\right) u(t) d t
$$

by

$$
D A=i H .
$$

(The notation $f$ is used to indicate a Cauchy principal value integral.) It is a simple exercise in the calculus of residues to show

$$
H e_{m}=\operatorname{sign}(m) e_{m}, \quad m \in \mathbb{Z},
$$

where

$$
\operatorname{sign}(m)=\left\{\begin{aligned}
-1 & \text { for } m<0 \\
0 & \text { for } m=0 \\
1 & \text { for } m>0
\end{aligned}\right.
$$


and from this one can see why the following well-known results hold; cf. Theorem 2.1 .

THEOREM 3.1. The Hilbert transform is a bounded operator

$$
H: L_{p}(\mathbb{T}) \rightarrow L_{p}(\mathbb{T}), \quad 1<p<\infty,
$$

and satisfies

$$
\begin{gathered}
(H u)_{m}^{\hat{m}}=\operatorname{sign}(m) \hat{u}_{m}, \quad m \in \mathbb{Z}, \\
H^{2} u=u-\frac{1}{2 \pi} \int_{-\pi}^{\pi} u d t .
\end{gathered}
$$

Since $A$ is a convolution operator,

$$
(A u)_{m}=\lambda_{m} \hat{u}_{m} \text { where } \lambda_{m}=\hat{\lambda}_{m},
$$

and using (2.6), (3.1), and Theorem 3.1, one can deduce

$$
\begin{gathered}
\lambda_{m}=1 /|m| \text { for } 0 \neq m \in \mathbb{Z}, \\
\lambda_{0}=\int_{-\pi}^{\pi} \Lambda(t) d t=2 \log 2, \\
A^{-1} f=-i H D f+\frac{1}{2 \pi \lambda_{0}} \int_{-\pi}^{\pi} f d t .
\end{gathered}
$$

Noting that $J^{s}$ commutes with convolution operators, the next theorem is now obvious. The notation $\rightleftarrows$ is used to indicate an isomorphism of Banach spaces.

THEOREM 3.2. For $1<p<\infty$ and $s \in \mathbb{R}$,

$$
A: H_{p}^{s}(\mathbb{T}) \rightleftarrows H_{p}^{s+1}(\mathbb{T}) \text {. }
$$

Define the vector operator

$$
\mathscr{A}(u, \alpha)=\left((A+K) u+\alpha, \int_{-\pi}^{\pi} u d t\right)
$$

and write the equations $(2.5)$ in the form

$$
\mathscr{A}(u, \alpha)=(f, \beta)
$$

then the main result for this section can be stated as follows.

THEOREM 3.3. For $1<p<\infty$ and $s \in \mathbb{R}$,

$$
\mathscr{A}: H_{p}^{s}(\mathbb{T}) \times \mathbb{K} \rightleftarrows H_{p}^{s+1}(\mathbb{T}) \times \mathbb{K} .
$$

Proof. Decompose $\mathscr{A}$ onto $\mathscr{A}=\mathscr{A}_{0}+\mathscr{X}$ where $\mathscr{A}_{0}(u, \alpha)=(A u, \alpha)$ and $\mathscr{K}(u, \alpha)$ $=\left(K u+\alpha, \int_{-\pi}^{\pi} u d t-\alpha\right)$; then $\mathscr{A}_{0}$ is an isomorphism by Theorem 3.2 and $\mathscr{X}$ is compact by (2.4). Therefore $\mathscr{A}$ is a Fredholm operator with zero index and we have only to observe that Theorem 1.1 implies $\mathscr{A}$ is one-one.

Remark 3.4. An alternative proof of the preceding theorem for the case $p=2$ is given by Hsiao and Wendland [7].

4. The Numerical Method. Denote the space of trigonometric polynomials of degree $\leqslant n$ by

$$
\mathscr{T}_{n}=\operatorname{span}\left\{e_{m}:|m| \leqslant n\right\}, \quad n=1,2,3, \ldots,
$$

and write

$$
(f \mid g)=\int_{-\pi}^{\pi} \overline{f(t)} g(t) d t
$$


then look for an approximate solution $\left(u_{n}, \alpha_{n}\right) \in \mathscr{T}_{n} \times \mathbb{K}$ satisfying the Galerkin equations

$$
\begin{gathered}
\left(\varphi \mid(A+K) u_{n}+\alpha_{n}\right)=(\varphi \mid f) \quad \text { for all } \varphi \in \mathscr{T}_{n}, \\
\int_{-\pi}^{\pi} u_{n} d t=\beta .
\end{gathered}
$$

This is a spectral method, because the eigenvalues and eigenvectors of $A$ are given by

$$
A e_{m}=\lambda_{m} e_{m}, \quad m \in \mathbb{Z}
$$

Write $u_{n}$ in the form

$$
u_{n}=\sum_{|m| \leqslant n} z_{m} \frac{e_{m}}{\lambda_{m}}
$$

then the unknowns $z_{-n}, \ldots, z_{n}, \alpha_{n}$ satisfy the $(2 n+2) \times(2 n+2)$ linear algebraic system

$$
\begin{gathered}
\sum_{|m| \leqslant n}\left[\delta_{l m}+\frac{1}{\lambda_{m}}\left(e_{l} \mid K e_{m}\right)\right] z_{m}+2 \pi \delta_{l 0} \alpha_{n}=\left(e_{l} \mid f\right), \quad|l| \leqslant n, \\
\frac{2 \pi}{\lambda_{0}} z_{0}=\beta .
\end{gathered}
$$

The inclusion of the factor $1 / \lambda_{m}$ in the $m$ th term of the expansion (4.2) for $u_{n}$ ensures that the $l_{2}$ condition number of the coefficient matrix is bounded as $n \rightarrow \infty$; cf. Mikhlin [14, Chapter 5].

Let the operator $P_{n}: L_{2}(\mathbb{T}) \rightarrow \mathscr{T}_{n}$ be the orthogonal projection onto the subspace $\mathscr{T}_{n}$; then Eq. (4.1a) is equivalent to

$$
P_{n}\left[(A+K) u_{n}+\alpha_{n}\right]=P_{n} f .
$$

Since

$$
P_{n} u=\frac{1}{2 \pi} \sum_{|m| \leqslant n} \hat{u}_{m} e_{m}
$$

is just a truncation of the Fourier series for $u$, it is clear that $P_{n}$ commutes with $A$ and satisfies $P_{n} 1=1$. Hence, if we define the operator

$$
\mathscr{A}_{n}(u, \alpha)=\left(\left(A+P_{n} K\right) u+\alpha, \int_{-\pi}^{\pi} u d t\right)
$$

then the Galerkin equations (4.1) can be written

$$
\mathscr{A}_{n}\left(u_{n}, \alpha_{n}\right)=\left(P_{n} f, \beta\right) \text {. }
$$

This form is convenient for carrying out the error analysis which follows.

THEOREM 4.1. If $1<p<\infty$ and $s \in \mathbb{R}$, then for $n$ sufficiently large, the solutions to (3.2) and (4.4) satisfy

$$
\left\|u_{n}-u\right\|_{H_{p}^{s}(\mathbf{T})}+\left|\alpha_{n}-\alpha\right| \leqslant c_{s, p}\left\|\left(I-P_{n}\right) u\right\|_{H_{p}^{s}(\mathbf{T})} .
$$

Proof. First note that

$$
\left(\mathscr{A}_{n}-\mathscr{A}\right)(u, \alpha)=\left(\left(P_{n}-I\right) K u, 0\right)
$$


then observe that, since $K$ has a smooth kernel,

$$
\lim _{n \rightarrow \infty}\left\|\left(I-P_{n}\right) K: H_{p}^{s}(\mathbb{T}) \rightarrow H_{p}^{s+1}(\mathbb{T})\right\|=0 .
$$

(This can easily be proved using Theorem 4.2 below.) Hence $\mathscr{A}_{n}$ converges to $\mathscr{A}$ in the operator norm and the stability result

$$
\left\|\mathscr{A}_{n}^{-1}: H_{p}^{s+1}(\mathbb{T}) \times \mathbb{K} \rightarrow H_{p}^{s}(\mathbb{T}) \times \mathbb{K}\right\| \leqslant c_{s, p}
$$

follows immediately from Theorem 3.3. The error estimate is now a consequence of the identity

$$
\mathscr{A}_{n}\left(u_{n}-u, \alpha_{n}-\alpha\right)=\left(\left(P_{n}-I\right) A u, 0\right)
$$

and the inequality (cf. Theorem 3.2)

$$
\left\|\left(I-P_{n}\right) A u\right\|_{H_{p}^{s+1}(\mathbf{T})}=\left\|A\left(I-P_{n}\right) u\right\|_{H_{p}^{s+1}(\mathbf{T})} \leqslant c\left\|\left(I-P_{n}\right) u\right\|_{H_{p}^{s}(\mathbf{T})} .
$$

The next step is to estimate the right-hand side of (4.5).

THEOREM 4.2. For $1<p<\infty$ and $-\infty<s<r<\infty$,

$$
\left\|\left(I-P_{n}\right) u\right\|_{H_{p}^{s}(\mathbf{T})} \leqslant c_{p, r-s} n^{s-r}\|u\|_{H_{p}^{r}(\mathbf{T})} .
$$

Proof. Since $J^{s}$ commutes with $P_{n}$ we may assume $s=0$. Given $n \geqslant 1$, let $l \geqslant 0$ be the unique integer satisfying $2^{l} \leqslant n<2^{l+1}$; then by the Marcinkiewicz Multiplier Theorem 2.1,

$$
\left\|\left(I-P_{n}\right) u\right\|_{p}=\frac{1}{2 \pi}\left\|\sum_{|m|>n} \hat{u}_{m} e_{m}\right\|_{p} \leqslant c_{p}\left\|\sum_{|m| \geqslant 2^{\prime}} \hat{u}_{m} e_{m}\right\|_{p} .
$$

The triangle inequality implies

$$
\begin{aligned}
\left\|\sum_{|m| \geqslant 2^{\prime}} \hat{u}_{m} e_{m}\right\|_{p} & \leqslant \sum_{j=l}^{\infty}\left\|\sum_{2^{j} \leqslant|m|<2^{j+1}} \hat{u}_{m} e_{m}\right\|_{p} \\
& \leqslant\left(\sum_{j=l}^{\infty} 2^{-j r}\right)\left(\sup _{j \geqslant l} 2^{j r}\left\|_{2^{j} \leqslant j m \mid<2^{j+1}} \hat{u}_{m} e_{m}\right\|_{p}\right),
\end{aligned}
$$

and another application of Theorem 2.1 gives

$$
\left\|\sum_{2^{j} \leqslant|m|<2^{j+1}} 2^{j r} \hat{u}_{m} e_{m}\right\|_{p} \leqslant c_{p, r}\left\|J^{r} u\right\|_{p} .
$$

By the choice of $l$,

$$
\sum_{j=1}^{\infty} 2^{-j r}=\frac{2^{-l r}}{1-2^{-r}} \leqslant\left(\frac{2^{r}}{1-2^{-r}}\right) n^{-r}
$$

and therefore

$$
\left\|\left(I-P_{n}\right) u\right\|_{p} \leqslant c_{p, r} n^{-r}\|u\|_{H_{p}^{r}(\mathbf{T})} .
$$

Remark 4.3. With minor modifications, the preceding proof actually establishes the slightly stronger result

$$
\left\|\left(I-P_{n}\right) u\right\|_{p} \leqslant c_{r, p} n^{-r}\|u\|_{B_{p \infty}^{r}(\mathbf{T})} .
$$


This estimate involving the Besov space $B_{p \infty}^{r}(\mathbb{T})$ is in a certain sense the best possible; cf. Bergh and Löfström [2, p. 188].

5. Exponential Rates of Convergence. The error estimates of the last section can sometimes be improved upon by making use of the Hilbert space

$$
X_{s, \varepsilon}, \quad s \in \mathbb{R}, \varepsilon>0,
$$

which is defined abstractly as the completion in the norm

$$
\|u\|_{s, \varepsilon}=\left(\sum_{m \in \mathbf{Z}}\left(1+|m|^{2}\right)^{s} \varepsilon^{2|m|}\left|\hat{u}_{m}\right|^{2}\right)^{1 / 2}
$$

of the space $\bigcup_{n=0}^{\infty} \mathscr{T}_{n}$ of trigonometric polynomials. These spaces are taken from the paper of Arnold [1], where a discussion of their basic properties may be found.

One can see from (2.6) that

$$
X_{s, \varepsilon} \subset C^{\infty}(\mathbb{T}) \text { for } s \in \mathbb{R} \text { and } \varepsilon>1,
$$

and from Parseval's identity (2.7) that

$$
X_{s, 1}=H_{2}^{s}(\mathbb{T}) \text { for } s \in \mathbb{R},
$$

with equivalence of norms. On the other hand, for $0<\varepsilon<1$, the space $X_{s, \varepsilon}$ contains elements which are not even distributions on $\mathbb{T}$. (If $u$ is a distribution, then $\hat{u}_{m}=O\left(|m|^{s}\right)$ for some integer $s>0$.) For our purposes, the utility of $X_{s, \varepsilon}$ stems from the following approximation theorem; cf. Theorem 4.2.

TheOREM 5.1. For $-\infty<s \leqslant r<\infty$ and $\varepsilon \geqslant 1$,

$$
\left\|\left(I-P_{n}\right) u\right\|_{H_{2}^{s}(\mathbf{T})} \leqslant \frac{1}{2 \pi} n^{s-r} \varepsilon^{-n}\|u\|_{r, \varepsilon} .
$$

Proof. Using Parseval's identity (2.7),

$$
\begin{aligned}
(2 \pi)^{2} & \left\|\left(I-P_{n}\right) u\right\|_{H_{2}^{s}(\mathbf{T})}^{2}=\sum_{|m|>n}\left(1+|m|^{2}\right)^{s}\left|\hat{u}_{m}\right|^{2} \\
& \leqslant\left(\sup _{|m|>n}\left(1+|m|^{2}\right)^{s-r} \varepsilon^{-2|m|}\right)\left(\sum_{|m|>n}\left(1+|m|^{2}\right)^{r} \varepsilon^{2|m|}\left|\hat{u}_{m}\right|^{2}\right) \\
& \leqslant\left(n^{s-r} \varepsilon^{-n}\right)^{2}\|u\|_{r, \varepsilon}^{2} .
\end{aligned}
$$

To make use of this result in Theorem 4.1, we must know $u \in X_{s, \varepsilon}$ for some $\varepsilon>1$, and hence the remainder of this section is devoted to establishing the necessary regularity theorem.

For $\delta>0$, let $Y_{\delta}$ be the vector space of functions $f$ which are analytic and satisfy $f(z+2 \pi)=f(z)$ in the strip $|\operatorname{Im}(z)|<\delta$. By Cauchy's theorem, if $f \in Y_{\delta_{0}}$, then

$$
\left|\hat{f}_{m}\right| \leqslant e^{-\delta|m|} \max _{\operatorname{Im}(z)= \pm \delta}|f(z)|, \quad 0 \leqslant \delta<\delta_{0}
$$

which shows

$$
Y_{\delta} \subset X_{s, \varepsilon} \text { for } s \in \mathbb{R} \text { and } 0<\varepsilon<e^{\delta} .
$$

We shall now assume:

the functions $\gamma_{1}, \gamma_{2}$ and $f$ appearing in (2.1) and (2.2) all belong to $Y_{\delta_{0}}$ for some $\delta_{0}>0$. 
This will enable us to prove the following

LEMMA 5.2. The operator

$$
K: X_{s_{1}, \varepsilon_{1}} \rightarrow X_{s_{2}, \varepsilon_{2}}
$$

is compact for $s_{1}, s_{2} \in \mathbb{R}, \varepsilon_{1}>e^{-\delta_{0}}$ and $0<\varepsilon_{2}<e^{\delta_{0}}$.

Proof. First we remark that since $u \mapsto\left(\left(1+|m|^{2}\right)^{s / 2} \varepsilon^{|m|} \hat{u}_{m}\right)_{m \in Z}$ is an isometry $X_{s, \varepsilon} \rightarrow l_{2}(\mathbb{Z})$, a subset $\mathscr{S}$ of $X_{s, \varepsilon}$ is precompact in $X_{s, \varepsilon}$ if and only if it is bounded and satisfies

$$
\lim _{n \rightarrow \infty} \sup _{u \in \mathscr{S}} \sum_{|m|>n}\left(1+|m|^{2}\right)^{s} \varepsilon^{2|m|}\left|\hat{u}_{m}\right|^{2}=0 ;
$$

cf. Edwards [4, p. 269]. The Fourier coefficients of the kernel of $K$,

$$
\hat{k}_{m l}=\int_{-\pi}^{\pi} \int_{-\pi}^{\pi} e^{-i(m x+l t)} k(x, t) d x d t
$$

satisfy

$$
\left|\hat{k}_{l m}\right| \leqslant e^{-\delta(|m|+|l|)} \max _{\operatorname{Im}\left(z_{1}\right)= \pm \delta, \operatorname{Im}\left(z_{2}\right)= \pm \delta}\left|k\left(z_{1}, z_{2}\right)\right|
$$

for $0 \leqslant \delta<\delta_{0}$; cf. (5.1) and [1, Lemma 4.5]. Choose $\delta$ so that $\varepsilon_{2} e^{-\delta}<1<\varepsilon_{1} e^{\delta}$; then by the Schwarz inequality,

$$
\begin{aligned}
\left|(K u)_{m}\right|^{2} & =\left|\sum_{l \in \mathbf{Z}} \hat{k}_{m,-l} \hat{u}_{l}\right|^{2} \\
& \leqslant c e^{-2 \delta|m|}\left(\sum_{l \in \mathbf{Z}}\left(1+|l|^{2}\right)^{-s_{1}}\left(\varepsilon_{1} e^{\delta}\right)^{-2|l|}\right)\left(\left.\sum_{l \in \mathbf{Z}}\left(1+|l|^{2}\right)^{s_{1}} \varepsilon_{1}^{2|l| \mid} \hat{u}_{l}\right|^{2}\right) \\
& =c e^{-2 \delta|m|}\|u\|_{s_{1}, \varepsilon_{1}}^{2},
\end{aligned}
$$

and hence

$$
\sup _{\|u\|_{s_{1}, f_{1}} \leqslant 1} \sum_{|m|>n}\left(1+|m|^{2}\right)^{s_{2}} \varepsilon_{2}^{2 \mid m \|}\left|(K u)_{m}\right|^{2} \leqslant \sum_{|m|>n}\left(1+|m|^{2}\right)^{s_{2}}\left(\varepsilon_{2} e^{-\delta}\right)^{2|m|} .
$$

This shows that $K$ maps the unit ball of $X_{s_{1}, \varepsilon_{1}}$ into a precompact subset of $X_{s_{2}, \varepsilon_{2}}$.

THEOREM 5.3. If (5.2) holds, then

$$
\mathscr{A}: X_{s, \varepsilon} \times \mathbb{K} \rightleftarrows X_{s+1, \varepsilon} \times \mathbb{K}
$$

for $s \in \mathbb{R}$ and $e^{-\delta_{0}}<\varepsilon<e^{\delta_{0}}$.

Proof. One has only to modify the proof of Theorem 3.3, noting that

$$
K: X_{s, \varepsilon} \rightarrow X_{s+1, \varepsilon}
$$

is compact by Lemma 5.2, and

$$
A: X_{s, \varepsilon} \rightleftarrows X_{s+1, \varepsilon} .
$$

Remark 5.4. Arnold [1] proved this theorem only for $e^{-\delta_{0}}<\varepsilon \leqslant 1$, which is not the case of interest to us.

6. Conclusion. Corresponding to the numerical solution $u_{n}$ on $\mathrm{T}$, there is a unique function $v_{n}$ on $\Gamma$ satisfying $u_{n}(t)=v_{n}[\gamma(t)]|\dot{\gamma}(t)|$; cf. (2.2). The resulting single-layer 
potential

$$
U_{n}(X)=\frac{1}{\pi} \int_{\Gamma} \log \left(\frac{1}{|X-Y|}\right) v_{n}(Y) d \sigma(Y)+\alpha_{n}, \quad X \in \mathbb{R}^{2},
$$

furnishes an approximation to $U$ which satisfies the following asymptotic error estimates.

THEOREM 6.1. Let $\alpha=\left(\alpha_{1}, \alpha_{2}\right)$ be any pair of nonnegative integers and write

$$
k=\alpha_{1}+\alpha_{2}, \quad \partial^{\alpha}=\left(\frac{\partial}{\partial X_{1}}\right)^{\alpha_{1}}\left(\frac{\partial}{\partial X_{2}}\right)^{\alpha_{2}} .
$$

(i) If $\Gamma$ and $g$ are $C^{\infty}$, then

$$
\left\|\partial^{\alpha}\left(U_{n}-U\right)\right\|_{L_{\infty}\left(\mathbf{R}^{2} \backslash \Gamma\right)} \leqslant c_{k, r} n^{k+1 / 2-r} \sqrt{\log n}\|g\|_{H_{2}^{r}(\Gamma)}
$$

for every $r>k+\frac{1}{2}$.

(ii) If $\Gamma$ and $g$ are analytic as in (5.2), then

$$
\left\|\partial^{\alpha}\left(U_{n}-U\right)\right\|_{L_{x}\left(\mathbf{R}^{2} \backslash \Gamma\right)} \leqslant c_{k, r, \varepsilon} \varepsilon^{-n} n^{k+1 / 2-r} \sqrt{\log n}\|g\|_{r, \varepsilon}
$$

for $r>k+\frac{1}{2}$ and $1 \leqslant \varepsilon<e^{\delta_{0}}$.

Proof. Put $g_{n}=U_{n} \mid \Gamma$; then the error $E_{n}=U_{n}-U$ satisfies

$$
\begin{array}{ll}
\Delta E_{n}=0 & \text { on } \mathbb{R}^{2} \backslash \Gamma, \\
E_{n}=g_{n}-g & \text { on } \Gamma, \\
E_{n}(X)=O(1), & \text { as }|X| \rightarrow \infty,
\end{array}
$$

and so, using the arguments set out in Arnold [1, Theorem 5.4],

$$
\left\|\partial^{\alpha} E_{n}\right\|_{L_{\infty}\left(\mathbf{R}^{2} \backslash \Gamma\right)} \leqslant c_{k} \mu^{-1 / 2}\left\|g_{n}-g\right\|_{H_{2}^{k+\mu+1 / 2}(\Gamma)}
$$

for $0<\mu<1$. Theorems 3.3 and 4.1 imply

$$
\begin{aligned}
\left\|g_{n}-g\right\|_{H_{2}^{s}(\Gamma)} & \leqslant c_{s}\left(\left\|u_{n}-u\right\|_{H_{2}^{s-1}(\mathbf{T})}+\left|\alpha_{n}-\alpha\right|\right) \\
& \leqslant c_{s}\left\|\left(I-P_{n}\right) u\right\|_{H_{2}^{s-1}(\mathbf{T})} \leqslant c_{r, s} n^{s-r}\|g\|_{H_{2}^{r}(\Gamma)}
\end{aligned}
$$

for $r>s$, so

$$
\left\|\partial^{\alpha} E_{n}\right\|_{L_{\infty}\left(\mathbf{R}^{2} \backslash \Gamma\right)} \leqslant c_{r, k} \mu^{-1 / 2} n^{k+\mu+1 / 2-r}\|g\|_{H_{2}^{r}(\Gamma)},
$$

and putting $\mu^{-1}=\log n$ this proves part (i). For part (ii), use Theorems 5.1 and 5.3 to obtain

$$
\left\|g_{n}-g\right\|_{H_{2}^{s}(\Gamma)} \leqslant c_{s, r} n^{s-r} \varepsilon^{-n}\|g\|_{r, \varepsilon}
$$

for $s \leqslant r$ and $1 \leqslant \varepsilon<e^{\delta_{0}}$.

Remark 6.2. In part (i) of the above theorem we have not made full use of the results of Sections 3 and 4 but only of the case $p=2$. This restriction is due to the trace theorems and to a priori estimates used to prove (6.2). It is interesting to note, however, that the well-known formula for the normal derivative of a single-layer potential allows one to prove natural error estimates for the normal derivative of $U_{n}$ in $H_{p}^{s}(\Gamma)$ for $1<p<\infty$. Actually, if instead of $H_{p}^{s}$ one works with the Besov space $B_{p q}^{s}$, for which the relevant trace theorems and a priori estimates hold for $1<p<\infty$, 
then it is possible to show [12, Theorem 6.2]

$$
\left\|\partial^{\alpha}\left(U_{n}-U\right)\right\|_{L_{\infty}\left(\mathbf{R}^{2} \backslash \Gamma\right)} \leqslant c_{k, p, r} n^{k+1 / p-r}\|g\|_{B_{p \infty}^{r}(\Gamma)}
$$

where $r>k+1 / p$ and $1<p<\infty$. Notice that here there is no factor involving $\log n$, the reason being that one can use the sharp imbedding $B_{p 1}^{1 / p}(\mathbb{T}) \subset C(\mathbb{T})$, $1<p<\infty$, to obtain maximum-norm estimates.

In contrast to the analogous results of Arnold [1, Theorem 5.3], our error estimates for $U_{n}$ and its derivatives are valid uniformly up to the boundary and not just on compact subsets of $\mathbb{R}^{2} \backslash \Gamma$. Of course, in practice one must take account of additional errors arising from the use of numerical quadratures to evaluate the integrals defining $\left(e_{l} \mid K e_{m}\right)$ and $\left(e_{l} \mid f\right)$ in (4.3) and $U_{n}$ in (6.1). For a discussion of these matters, including the use of fast Fourier transforms, we refer to [9], [10, Section 5.4], and [11]. Results of numerical experiments are given in [5], [9], [11], [17].

Finally, I wish to thank Professor $\mathrm{H}$. Triebel for some discussions which were helpful in the proof of Theorem 4.2.

Department of Mathematics

Oregon State University

Corvallis, Oregon 97331-4605

1. D. N. ARNOLD, "A spline-trigonometric Galerkin method and an exponentially convergent boundary integral method," Math. Comp., v. 41, 1983, pp. 383-397.

2. J. BERGH \& L. LÖFSTRÖM, Interpolation Spaces, Springer-Verlag, Berlin and New York, 1976.

3. S. Christiansen, "On two methods for elimination of non-unique solutions of an integral equation with logarithmic kernel," Applicable Anal., v. 13, 1982, pp. 1-18.

4. R. E. EdwardS, Functional Analysis, Holt, Rinehart \& Winston, New York, 1965.

5. P. HeNRICI, "Fast Fourier methods in computational complex analysis," SIAM Rev., v. 21, 1979, pp. 481-527.

6. G. C. Hsiao, P. Kopp \& W. L. Wendland, "A Galerkin collocation method for some integral equations of the first kind," Computing, v. 25, 1980, pp. 89-130.

7. G. C. Hsiao \& W. L. Wendland, "A finite element method for some integral equations of the first kind," J. Math. Anal. Appl., v. 58, 1977, pp. 449-481.

8. M. A. JASWON \& G. T. SYmm, Integral Equation Methods in Potential Theory and Elastostatics, Academic Press, New York, 1977.

9. U. Lamp, K.-T. Schleicher \& W. L. Wendland, "The fast Fourier transform and the numerical solution of one-dimensional boundary integral equations," Numer. Math., v. 47, 1985, pp. 15-38.

10. W. MCLean, Boundary Integral Methods for the Laplace Equation, Thesis, Australian National University, Canberra, 1985.

11. W. MCLean, A Computational Method for Solving a First Kind Integral Equation, Research Report CMA-R15-85, Centre for Mathematical Analysis, Australian National University, 1985.

12. W. MCLEAN, "Error estimates for a first kind integral equation and an associated boundary value problem," Proc. Centre Math. Anal. Austral. Nat. Univ., v. 9, 1985, pp. 223-240.

13. J. Marcinkiewicz, "Sur les multiplicateurs des séries de Fourier," Studia Math., v. 8, 1939, pp. 78-91.

14. S. G. MikHLIN, The Numerical Performance of Variational Methods, Wolters-Noordhoff, Groningen, 1971.

15. S. M. NIKol'SKII, Approximation of Functions of Several Variables and Imbedding Theorems, Springer-Verlag, Berlin and New York, 1975.

16. G. VeRChOta, "Layer potentials and regularity for the Dirichlet problem for Laplace's equation in Lipschitz domains," J. Funct. Anal., v. 59, 1984, pp. 572-611.

17. R. Wegmann, "Convergence proofs and error estimates for an iterative method for conformal mapping," Numer. Math., v. 44, 1984, pp. 435-461. 